\title{
Bacterial-type oxygen detoxification and iron-sulfur cluster assembly in amoebal relict mitochondria
}

\author{
Barbora Maralikova, ${ }^{1}$ Vahab Ali, ${ }^{2, \dagger}$ \\ Kumiko Nakada-Tsukui, ${ }^{2,3}$ Tomoyoshi Nozaki ${ }^{2,3}$ \\ Mark van der Giezen, ${ }^{1, \neq}$ Katrin Henze ${ }^{4}$ and \\ Jorge Tovar ${ }^{1}$ \\ ${ }^{1}$ School of Biological Sciences, Royal Holloway \\ University of London, Egham TW2O OEX, UK. \\ ${ }^{2}$ Department of Parasitology, Gunma University \\ Graduate School of Medicine, Maebashi, 371-8 511, \\ Japan. \\ ${ }^{3}$ Department of Parasitology, National Institute of \\ Infectious Diseases, Tokyo 162-8640, Japan. \\ ${ }^{4}$ Institute of Botany III, Heinrich Heine University, 40225 \\ Düsseldorf, Germany.
}

\section{Summary}

The assembly of vital reactive iron-sulfur (Fe-S) cofactors in eukaryotes is mediated by proteins inherited from the original mitochondrial endosymbiont. Uniquely among eukaryotes, however, Entamoeba and Mastigamoeba lack such mitochondrial-type Fe-S cluster assembly proteins and possess instead an analogous bacterial-type system acquired by lateral gene transfer. Here we demonstrate, using immunomicroscopy and biochemical methods, that beyond their predicted cytosolic distribution the bacterial-type Fe-S cluster assembly proteins NifS and NifU have been recruited to function within the relict mitochondrial organelles (mitosomes) of Entamoeba histolytica. Both Nif proteins are 10-fold more concentrated within mitosomes compared with their cytosolic distribution suggesting that active $\mathrm{Fe}-\mathrm{S}$ protein maturation occurs in these organelles. Quantitative immunoelectron microscopy showed that amoebal mitosomes are minute but highly abundant cellular structures that occupy up to $2 \%$ of the total cell volume. In addition, protein colocalization studies

Received 8 July, 2009; revised 13 October, 2009; accepted 15 October, 2009. *For correspondence. E-mail j.tovar@ rhul.ac.uk; Tel. (+44) 1784 414159; Fax (+44) 1784414224.

Present addresses: ${ }^{\dagger}$ Department of Biochemistry, Rajendra Memorial Research Institute of Medical Sciences, Agamkuan, Patna-800 007, India; ${ }^{\ddagger}$ Centre for Eukaryotic Evolutionary Microbiology, School of Biosciences, University of Exeter, Exeter, EX4 4QD, UK. allowed identification of the amoebal hydroperoxide detoxification enzyme rubrerythrin as a mitosomal protein. This protein contains functional Fe-S centres and exhibits peroxidase activity in vitro. Our findings demonstrate the role of analogous protein replacement in mitochondrial organelle evolution and suggest that the relict mitochondrial organelles of Entamoeba are important sites of metabolic activity that function in Fe-S proteinmediated oxygen detoxification.

\section{Introduction}

Life on earth is dependent on the activities of highly reactive Fe-S centre-containing proteins that mediate key biological functions such as electron transport, metabolic regulation, metalloenzyme catalysis and chemical sensing (Beinert et al., 1997; Beinert, 2000). In bacteria such Fe-S centres are assembled via one or more of three systems of diverse molecular complexity which are known as nitrogen fixation (Nif), mobilization of sulfur and iron-sulfur cluster (Isc) systems (Lill and Kispal, 2000; Rees and Howard, 2000; Craig and Marszalek, 2002; Takahashi and Tokumoto, 2002; Frazzon and Dean, 2003; Loiseau et al., 2003; Outten et al., 2003; Lill and Mühlenhoff, 2008). Although variable in their molecular composition all three $\mathrm{Fe}-\mathrm{S}$ cluster assembly systems rely on the enzymatic transfer of molecular sulfur from cysteine to molecular iron through the concerted action of cysteine desulfurase and of scaffold iron-binding proteins to form transient Fe-S centres, which are then transferred to their final target apoproteins in a process known as $\mathrm{Fe}-\mathrm{S}$ protein maturation.

Most eukaryotic cells inherited the Isc system from the original mitochondrial endosymbiont. Genetic and biochemical studies have shown that the maturation of cytosolic and mitochondrial Fe-S proteins is dependent on $\mathrm{Fe}-\mathrm{S}$ centres assembled in mitochondria and have identified Fe-S cluster assembly as the only essential biosynthetic function of this organelle (Lill and Kispal, 2000; Gerber et al., 2004). Interestingly, microbial eukaryotes that lack recognizable mitochondria seem to carry out this function in mitochondrion-related organelles known as mitosomes or hydrogenosomes (Tovar et al., 2003; Suták et al., 2004; Goldberg et al., 2008). These organelles share a common ancestry with mitochondria and have 
evolved several times independently in diverse protist and fungal lineages (Embley et al., 1995; van der Giezen et al., 2005a; Embley, 2006; Tovar, 2007). Given the monophyletic nature of mitochondria and the mutually exclusive distribution between mitosomes, mitochondria and hydrogenosomes, all of these organelles are considered evolutionary derivatives of the original mitochondrial endosymbiont.

The functional minimalism of mitosomes is apparent from genome surveys of mitosome-bearing organisms such as Giardia, Entamoeba and Encephalitozoon which have revealed molecular chaperonins and Fe-S cluster assembly proteins as sole common features (Katinka et al., 2001; Loftus et al., 2005; Morrison et al., 2007). Fe-S cluster biosynthesis and Fe-S protein maturation were originally demonstrated in Giardia mitosomes (Tovar et al., 2003) and recent evidence on the localization and functionality of microsporidial Fe-S cluster assembly proteins suggests that Encephalitozoon cuniculi mitosomes may also fulfil this function (Goldberg et al., 2008). The essential nature of Fe-S cluster assembly in eukaryotes and the lack of evidence for additional mitosomal functions strongly suggest that retention of mitochondrial organelles may be driven by the need of in organello Fe-S cluster assembly and Fe-S protein maturation (Embley et al., 2003; Tovar et al., 2003). However, the discovery that Entamoeba lacks the mitochondrial-type Isc system of Fe-S cluster assembly and possesses instead a non-redundant bacterial-type Nif system acquired by lateral transfer from epsilon proteobacteria (Ali et al., 2004; van der Giezen et al., 2004) challenged the validity of this hypothesis. Here we report on the unusual cellular distribution of the amoebal Fe-S cluster assembly proteins NifS and NifU and show that peroxide detoxification and FeS cluster assembly are physiological functions of Entamoeba histolytica mitosomes.

\section{Results}

\section{Dual distribution of amoebal NifS/NifU}

Amoebas of the genera Entamoeba and Mastigoamoeba stand alone among eukaryotes in possessing bacterialtype Fe-S cluster assembly proteins instead of their canonical mitochondrial-type analogues (Ali et al., 2004; van der Giezen et al., 2004; Gill et al., 2007). We previously demonstrated that $E$. histolytica NifS and NifU were likely acquired by lateral gene transfer from epsilonproteobacteria and that these proteins fulfil a nonredundant functional role (Ali et al., 2004; van der Giezen et al., 2004). Coexpressed NifS and NifU are able to complement the growth defect of mutant Escherichia coli whose isc and sufoperons had been removed by targeted gene deletion, demonstrating that both proteins are necessary and sufficient for the biosynthesis of Fe-S clusters under anaerobic conditions (Ali et al., 2004). Purified recombinant proteins NifS and NifU expressed in bacteria were used to generate specific homologous antibodies which have been used in this study to define the cellular distribution of these proteins in parasite trophozoites.

Laser scanning confocal microscopy imaging revealed an unconventional distribution pattern for amoebal NifS and NifU. Both proteins appear distributed throughout the cytoplasm but their distribution seems uneven, with some punctate labelling that could suggest partial compartmentalization (Fig. 1A-H). Western blot analysis of trophozoite extracts separated by differential centrifugation showed that although most amoebal Nif proteins remain in the high speed supernatant (cytosol), a significant proportion of each protein appears associated with the mixed membrane fraction (MMF), which is a mixture of broken membranes and membrane-bounded organelles (Fig. 1I). Together these data suggested that E. histolytica Nif proteins could be both cytosolic and associated with membranous cellular structures.

\section{NifS and NifU are enriched in mitosomes}

To investigate the apparent unusual distribution of Nif proteins further we used a more refined and powerful imaging method, immunoelectron microscopy. Our initial studies focused on the identification of Entamoeba mitosomes using a specific antibody against the mitosomal marker protein Cpn60, a crucial control in our experiments because the Fe-S cluster biosynthetic proteins in all other eukaryotes are known to reside in mitochondrial organelles (including mitosomes and hydrogenosomes where present). Immunogold labelling of Cpn60 identified mitosomes as minute spherical structures of around $100 \mathrm{~nm}$ in diameter surrounded by two limiting membranes (Fig. 2A and D). Little or no cytosolic labelling was detected for this antigen, in agreement with the distribution of Cpn60 observed by confocal microscopy (León-Avila and Tovar, 2004). Interestingly, similar cellular organelles were observed by immunogold labelling of NifS and NifU. In this case, however, both antigens were also found widely distributed throughout the cytosol (Fig. 2B, C, E and F), in agreement with the distribution observed for these proteins by confocal imaging (Fig. 1). Double labelling for Cpn60 and NifS as well as Cpn60 and NifU colocalized these three antigens to the same intracellular structures (Fig. 2G and $\mathrm{H}$ respectively) thus demonstrating that amoebal Fe-S cluster assembly proteins have a dual cytosolic and compartmentalized distribution and that the compartments harbouring NifS and NifU are mitosomes.

\section{Ectopic expression of NifS and NifU}

In an attempt to increase the rate of Fe-S cluster assembly and Fe-S protein maturation in the parasite we generated

(C) 2009 Blackwell Publishing Ltd, Cellular Microbiology, 12, 331-342 

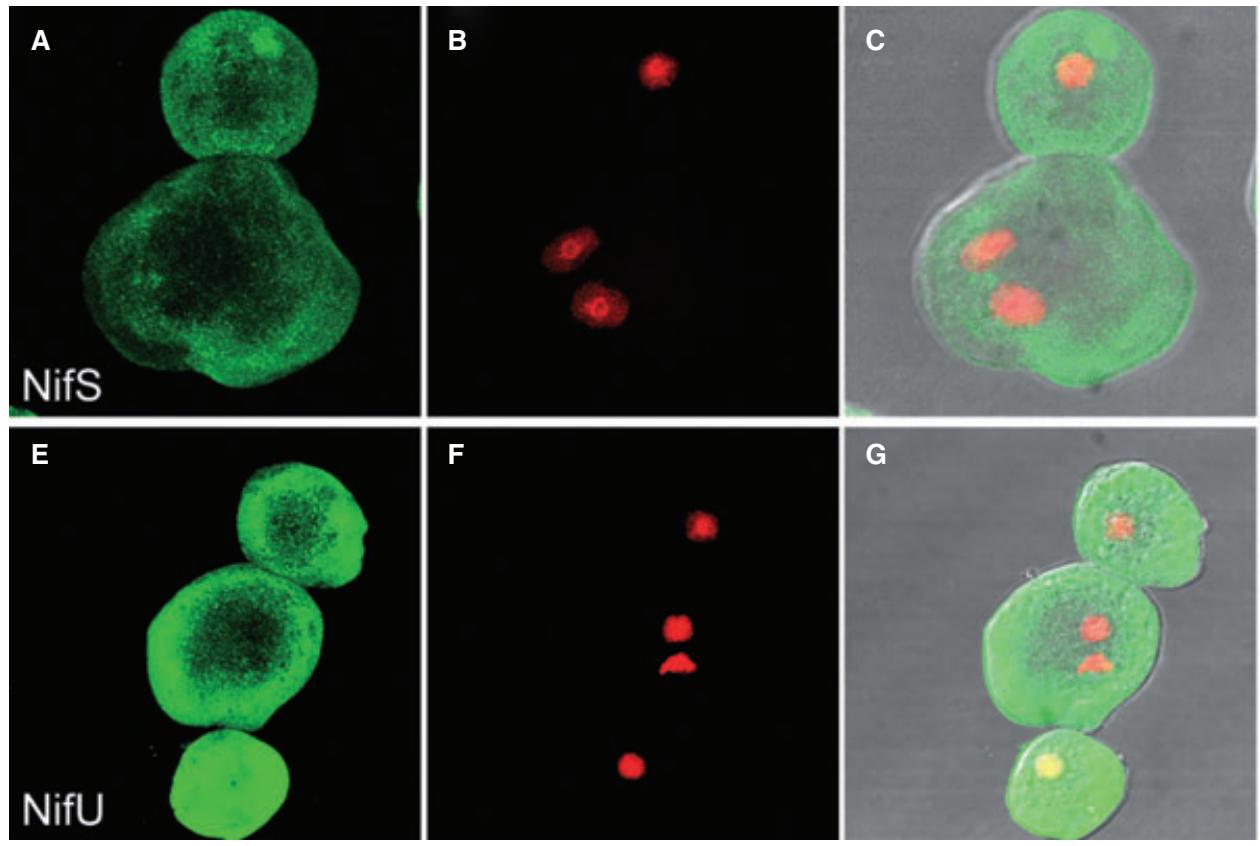
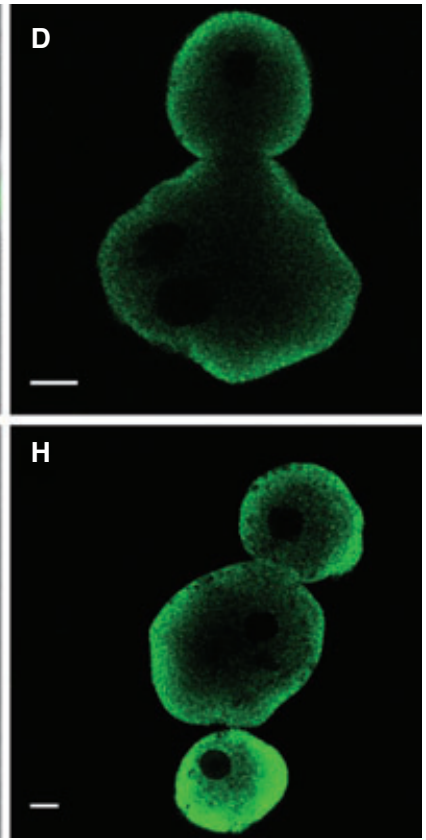

I

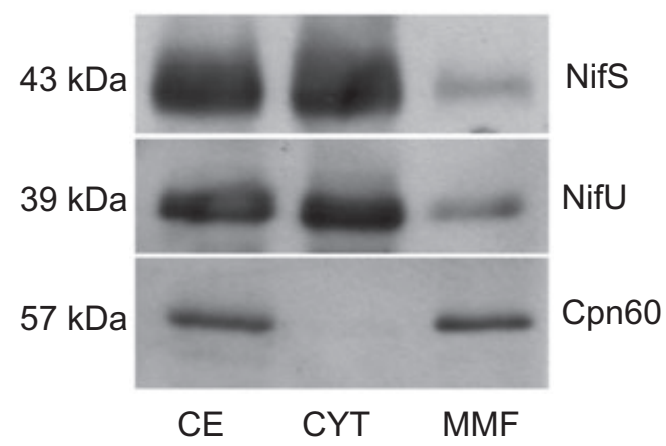

Fig. 1. Immunolocalization of E. histolytica Nif proteins by confocal microscopy and Western blotting. Fixed trophozoites were incubated with antibodies against EhNifS ( $A$ and $D$ ) or EhNifU ( $E$ and $H$ ) and post-treated with propidium iodide to identify nuclei (B and $F)$. Panels $A$ and $E$ show maximum projections of stacked optical slices $1 \mu \mathrm{m}$ in thickness for NifS and NifU respectively. Corresponding single optical slices are shown on $\mathrm{D}$ and $\mathrm{H}$; note that little or no antigen staining is apparent in the nucleus (compare bottom cell on $\mathrm{E}$ and $\mathrm{H}$ ). Panels $\mathrm{C}$ and $\mathrm{G}$ show antibody and propidium iodide staining superimposed on DIC images.

I. Parasite cell-free extracts were fractionated by differential centrifugation and analysed by Western blotting; the specificity of antibodies used and the molecular mass of identified proteins are indicated. CE, crude extract; CYT, cytosolic fraction; MMF, mixed membrane fraction. Scale bars: $5 \mu \mathrm{m}$.

transgenic E. histolytica lines ectopically expressing epitope-tagged variants of NifS and NifU either individually or simultaneously. Western blot analysis of transgenic parasite extracts demonstrated a 1.6-fold and a 2.6-fold overexpression of recombinant NifS and NifU respectively (Fig. S1). Confocal microscopy imaging confirmed that the overexpressed tagged variants colocalize with endogenous Nif proteins in transgenic trophozoites (data not shown but submitted for review), clearly indicating that the presence of epitope tags does not affect protein localization. Biochemical assays revealed a 1.5- and 2-fold enhancement of catalytic activity for the amoebal Fe-S proteins pyruvate : ferredoxin oxidoreductase and ferre- doxin (Fig. 3) suggesting a direct correlation between Nif protein levels and Fe-S protein maturation. Interestingly, these transgenic parasite lines also displayed a growth phenotype suggesting that the activity of Fe-S proteins and the level of Nif protein expression can be growth rate limiting (Fig. S2).

\section{Peroxide detoxification in mitosomes}

The discovery that Nif proteins accumulate in Entamoeba mitosomes and the previous demonstration that NifS and NifU are necessary and sufficient for Fe-S cluster assembly suggested the existence of at least one Fe-S protein in 


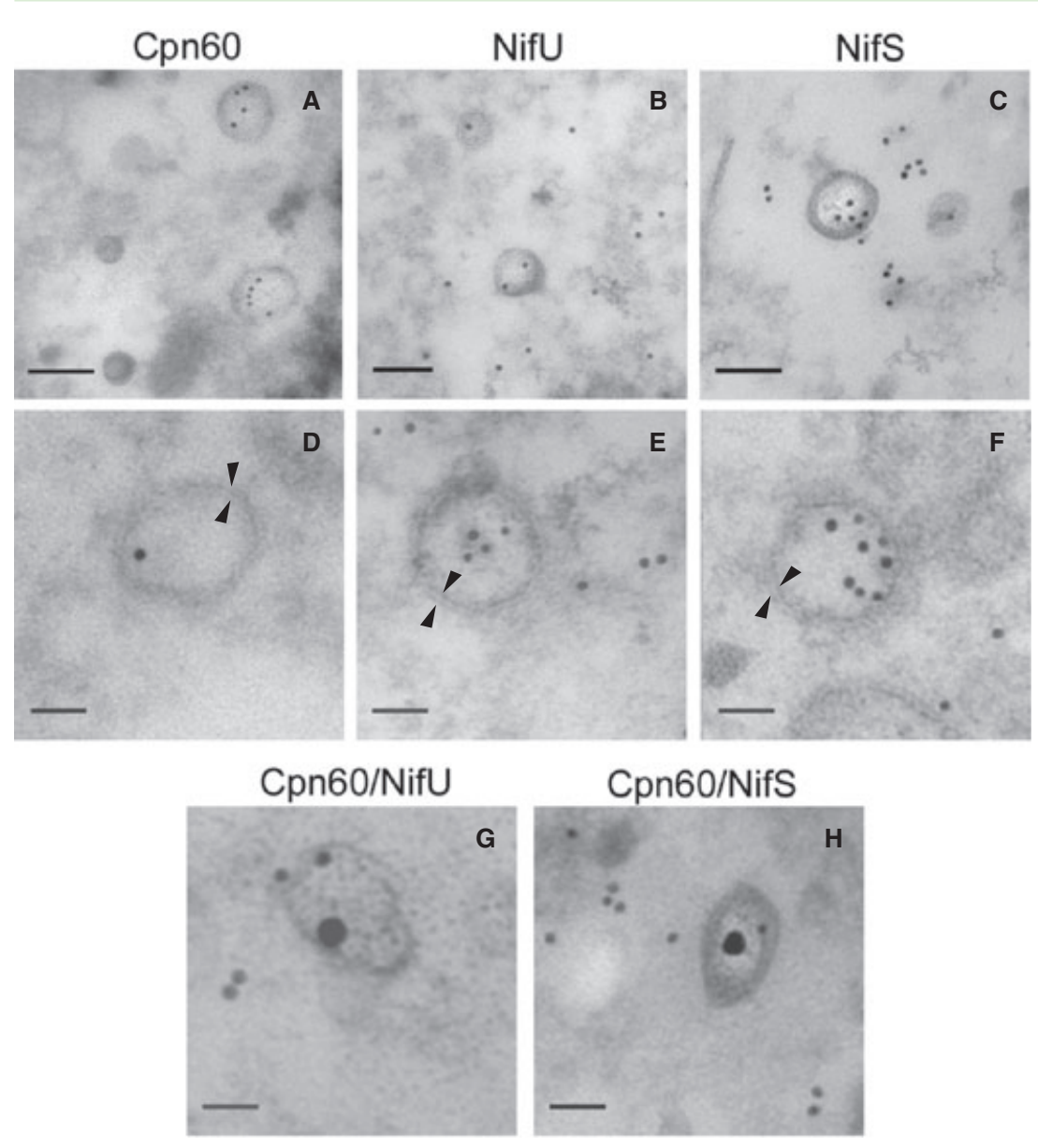

Fig. 2. Localization of amoebal NifS and NifU proteins by immunoelectron microscopy. Parasite trophozoites were fixed and embedded in resin as described in Experimental procedures. Ultra-thin sections were cut and mounted on grids before exposure to antibodies specific for EhCpn60 (A and D), EhNifU (B and E) or EhNifS (C and F). Double labelling using $20 \mathrm{~nm}$ gold particles for $\mathrm{Cpn60}$ and $10 \mathrm{~nm}$ particles for $\mathrm{Nif}$ proteins is shown in $\mathrm{G}$ and $\mathrm{H}$. Arrow heads highlight organellar membranes. Scale bars: $100 \mathrm{~nm}(\mathrm{~A}-\mathrm{C}), 50 \mathrm{~nm}(\mathrm{D}-\mathrm{F}, \mathrm{G}, \mathrm{H})$.

mitosomes that might require in organello maturation. Although none of the few known mitosomal proteins carries reactive Fe-S centres (Bakatselou et al., 2000; 2003; León-Avila and Tovar, 2004; Chan et al., 2005; van der Giezen et al., 2005b; Aguilera et al., 2008), the identification in the Entamoeba genome of a gene encoding rubrerythrin (Rbr) - a hydroperoxide detoxification Fe-S protein found predominantly in anaerobic bacteria but also recently discovered in Trichomonas vaginalis hydrogenosomes (Loftus et al., 2005; Pütz et al., 2005) - suggested $\mathrm{Rbr}$ as a putative mitosomal protein in E. histolytica.

Bacterial Rbr contains an Fe (S-Cys) $)_{4}$ reactive centre and a non-sulfur oxo-bridged diiron centre that are essential for functionality. All the cysteine, glutamate and histidine amino acid residues that form and hold these functional centres together are conserved in the amoebal $\mathrm{Rbr}$ homologue suggesting a functional amoebal peroxidase (Fig. 4A). We cloned and overexpressed this protein in a heterologous bacterial system. Affinity purified protein was then tested for functionality. NADPH-dependent peroxidase activity was observed when $E$. histolytica Rbr was added to an in vitro system containing NADPH, spinach ferredoxin : $\mathrm{NADP}^{+}$oxidoreductase, Clostridium pasteur- ianum rubredoxin (Rub) and $\mathrm{H}_{2} \mathrm{O}_{2}$ (Fig. 4B). This activity indicates that, analogous to prokaryotic $\mathrm{Rbr}$ and its $T$. vaginalis homologue, recombinant $E$. histolytica Rbr transfers electrons from Rubred to peroxide, a process where electrons initially enter Rbr via the $\mathrm{Fe}$ (SCys) ${ }_{4}$ centre and are then transferred to the diiron centre and on to peroxide (Coulter et al., 1999). With a specific activity of $120 \mathrm{nmol} \mathrm{min}{ }^{-1} \mathrm{mg}^{-1}$ (corresponding to $3 \mathrm{mM} \mathrm{min}^{-1} \mathrm{mM}^{-1}$ ) recombinant $E$. histolytica $\mathrm{Rbr}$ was similar to that of recombinant $T$. vaginalis $\mathrm{Rbr}\left(125 \mathrm{nmol} \mathrm{min} \mathrm{mg}^{-1}\right)$. Specific activity of Rbr from the archaebacterium Pyrococcus furiosus and that of the proteobacterium Desulfovibrio vulgaris are three times and 20 times higher respectively (Coulter and Kurtz., 2001; Weinberg et al., 2004). This difference may be due to the artificial electron-donor system in the in vitro assay, as Rub is the endogenous electron donor to $\mathrm{Rbr}$ in prokaryotes but is absent from the genomes of E. histolytica and T. vaginalis. In addition the His-tags at the C-termini of the recombinant eukaryotic proteins might interfere with dimerization, as the C-termini in active $\mathrm{Rbr}$ dimers lie at the subunit interface (Jin et al., 2002).

Specific antibodies generated against recombinant T. vaginalis $\mathrm{Rbr}$ were then used in cell fractionation and 
A

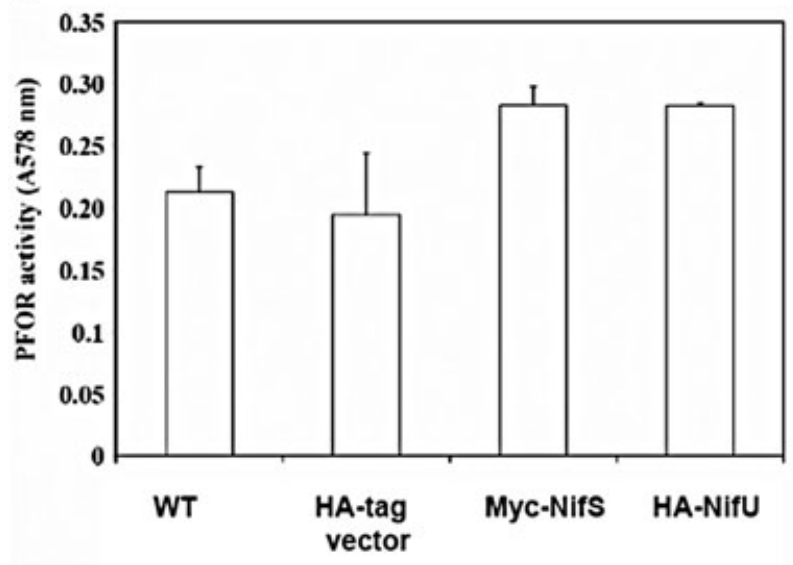

B

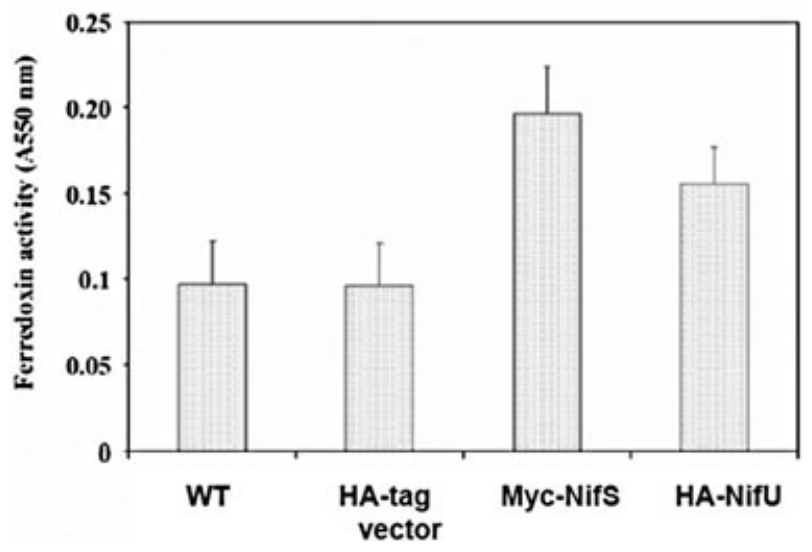

Fig. 3. Enhanced activity of $\mathrm{FeS}$ proteins in transgenic parasites by ectopic overexpression of amoebal NifS or NifU. Pyruvate: ferredoxin oxidoreductase (A) and ferredoxin (B) activities in E. histolytica trophozoites were measured in cell-free extracts as described in Experimental procedures. Bars represent the mean values and standard errors of data points collected in duplicate in at least two independent experiments. WT, untransfected parasites HA-tag vector, control parasites transfected with the empty HA-tag expression vector; Myc-NifS, parasites overexpressing myc-tagged NifS protein; HA-NifU, parasites overexpressing HA-tagged NifU protein. immunomicroscopy experiments to determine the intracellular distribution of amoebal Rbr. Confocal microscopy imaging demonstrated a punctuate distribution for this antigen in Entamoeba trophozites, revealing the presence of dozens of Rbr-containing compartments in every cell of the parasite population (Fig. 5A-E). Subcellular fractionation by differential centrifugation and separation of the MMF in sucrose density gradients identified a reactive antigen of the monomeric size predicted for $E$. histolytica Rbr in the high sucrose density fractions (Fig. 5F) further indicating the compartmentalization of Rbr in Entamoeba. Immunogold labelling of amoebal Rbr in ultra-thin transmission electron microscopy sections revealed the presence of highly abundant double membrane-bounded structures (Fig. 6A-D) of equivalent size and appearance to those observed with the anti-Cpn60 antibodies (Fig. 6E; see also Fig. 2). Double labelling for Rbr and Cpn60 localized both of these antigens in the same type of organelles demonstrating that E. histolytica Rbr is a mitosomal protein (Fig. 6F).

\section{Redefinition of Entamoeba mitosomes}

Considerable uncertainty had surrounded the ultrastructure of E. histolytica mitosomes (Aguilera et al., 2008) as previous attempts to localize Cpn60 in electron microscopy micrographs proved unsuccessful (Ghosh et al., 2000). Based on the immunoelectron microscopy data generated in our study it is now possible to define E. histolytica mitosomes as minute but abundant double membrane-bounded organelles housing the mitochondrial chaperonin Cpn60, Rbr and the Fe-S cluster assembly proteins NifS and NifU. At an estimated $100 \mathrm{~nm}$ in diameter and a mitosomal area of $7.9 \times 10^{-3} \mu \mathrm{m}^{2}$, Nif labelling densities were 10-fold higher in mitosomes than in the cytosol demonstrating a significant enrichment of $\mathrm{Fe}-\mathrm{S}$ cluster assembly proteins in these organelles (Table 1). Quantification in electron micrographs of labelled organelles allowed estimation of an average 34, 27, 37 and 29 mitosomes $\mu \mathrm{m}^{-3}$ for the NifS, NifU, Cpn60 and $\mathrm{Rbr}$ antigens respectively (see Experimental proce-

Table 1. Immunoelectron microscopy-based redefinition of E. histolytica mitosomes.

\begin{tabular}{|c|c|c|c|c|}
\hline \multirow[b]{2}{*}{ Parameter } & \multicolumn{4}{|c|}{ Antigen } \\
\hline & Cpn60 & NifU & NifS & $\mathrm{Rbr}$ \\
\hline \multicolumn{5}{|l|}{ Labelling density (golds $\left.\mu \mathrm{m}^{-2}\right)^{\mathrm{a}}$} \\
\hline Cytosol & $2.3 \pm 0.90$ & $34 \pm 5.2$ & $32 \pm 4.2$ & $1.9 \pm 1.1$ \\
\hline Mitosomes & $407 \pm 38$ & $357 \pm 49$ & $318 \pm 29$ & $306 \pm 43$ \\
\hline Mitosome : cytosol antigen distribution ratio & 177 & 11 & 10 & 161 \\
\hline Mitosomes $\mu \mathrm{m}^{-3}$ & 37 & 27 & 34 & 29 \\
\hline Mitosomes per trophozoite $\left(\times 10^{5}\right)$ & 1.54 & 1.13 & 1.42 & 1.21 \\
\hline Percentage of total cell volume & 1.9 & 1.4 & 1.8 & 1.5 \\
\hline
\end{tabular}

a. Labelling densities and organelle quantification were determined as described in Experimental procedures ( $n=8$ for Cpn60, $n=10$ for NifU, $n=10$ for NifS and $n=10$ for Rbr; $n=$ number of scanned micrographs). 
E. histolytica

T. vaginalis

M. jannashii

A. fulgidus

P. gingivalis

D. vulgaris

E. histolytica

T. vaginalis

M. jannashii

A. fulgidus

P. gingivalis

D. vulgaris

E. histolytica

T. vaginalis

M. jannashii

A. fulgidus

P. gingivalis

D. vulgaris

E. histolytica

T. vaginalis

M. jannashii

A. fulgidus

P. gingivalis

D. vulgaris
----------MATLINLCKAFAGESQARNRYLIYAKTAKKEGLDVIAQLFNETAN ---n---MSLKGTQTEKNLACAFAGESMARNRYTFFAEVAKKQGYEQIAQLFIETAE ----MINNFFVINMKETLKNLTKAYIGESLARNRYTCYAKIAKQEGYEQIAEIFLLTAE

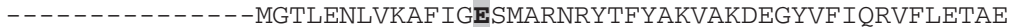
MSIKKKTEMNKSIKGSKTEKHLLMAFAGESQARSRYTFFASVAKKEGYEQIAGVFMETAE -------MKALKGTKTERNILTAFAGESQARNRYDYFGARAKKDGFVQIADIFAETAS * *************

QEGTHARILFEMIQSLKKE---GQETPKIE-TAVPIDFGTTADNLLAAIAGETYEHETMY NEKVHANYFWSQIK---G-----LGSVKIETEVPACGVNDTLTNLRNAAAGEYAEHTTDY NEREHAKWLYYLITELKKKYNIDDKA IKVDGVEVPIVLGNTAENLKASIEGEHFEHTEMY NEKEHAENLLKFIQQLRG----DNAAIKVE-AEAPLVWGTTVENLKAAIEGEHYENSEMY OEKEHAKRFFSFLE-------GGMLEITASFPAGIIGSTAENLRAAAAGENEEWTDLY QEKEHAKRLFKFLE--------GGEVEIVAAFPAGIIGDTHANLISSAAGENHEYTEMY * $* *$

PEFAKVAKEEGHAQIAARLNLIAKAELNHHNNYQKILDELKANSLYKKTEKVFWVCRECG PHFADVAEKEGFAKIAKAFRGIAAVEKEHEIRFNTLAKOVESSTVFKREAVVAWKCRNCG PKFADIAEKEGLKEIADRLRAIGIAEKHHEERFKKLLKEVEEGTVFKKDKPVEWVCRKCG PQFADEAEKEGFKDIADRLRAIGKAEEHHERRYRRLLAEVENGTFFKRDKEIAWVCLECG PAFAETAEEEGFKEIAAVFRQIAKVEAEHERRYLALLAHVEDGSVFERTEEIAWQCRNCG PSFARIAREEGFDEIAKVFMSIAVAEEFHERRFLGFAKNVKEARVFVRESSVVWRCRNCG ********** $*$ * YIFESTQPPKVCPLCGEPGDFFRVQVSI-YVVRAKVAPKACPVCFKPQGWFEIKEVLEFVHLGKEPPEKCPSCSHPRKYFEVKCEKYYIHYGTEPPEECPSCGHPKAYYVAEDLLSL YVITSKKAPKLCPACAHPQAYFEPMKTNYFLHEGNEAPHLCPACAHPQAHFELLGINW-

\section{B}

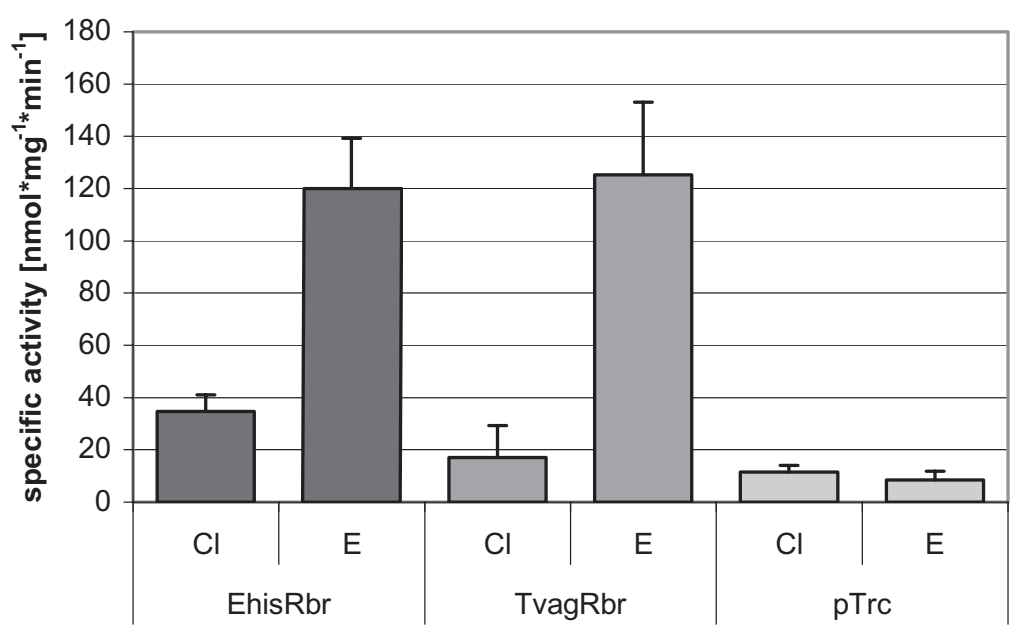

Fig. 4. Properties and functionality of $E$. histolytica rubrerythrin.

A. ClustalW alignment of eukaryotic rubrerythrin sequences with homologues from archaebacteria and eubacteria. Conserved glutamate and histidine residues holding the oxo-bridged diiron centre and cysteine residues involved in the Fe (S-Cys) ${ }_{4}$ reactive centre (Coulter et al., 1999) are shaded in grey.

B. Specific activity of recombinant His-tagged rubrerythrin from E. histolytica and T. vaginalis. pTrc, empty vector control; $\mathrm{Cl}$, cleared lysate; E, Ni-NTA purified protein.

dures for calculations). Although Entamoeba trophozoites are pleomorphic, the size of an average amoeba is around $20 \mu \mathrm{m}$ in diameter (Müller, 2000) and its average cell volume is $4189 \mu \mathrm{m}^{3}$. Thus an average trophozoite may contain over 100000 mitosomes, a hitherto unsuspected abundance for these organelles in protozoan parasites (Table 1). Further, at an average mitosomal volume of $5.2 \times 10^{-4} \mu \mathrm{m}^{3}$ the total volume occupied by these organelles in E. histolytica may range between 60 and $82 \mu \mathrm{m}^{3}$, representing approximately $1.4-1.9 \%$ of the total cell volume (Table 1 ). This figure represents only a fraction of the total cell volume occupied by other mitochondrial organelles (>10\% for mitochondria, 10\% for trichomonad hydrogenosomes) (Müller, 2000) but it 

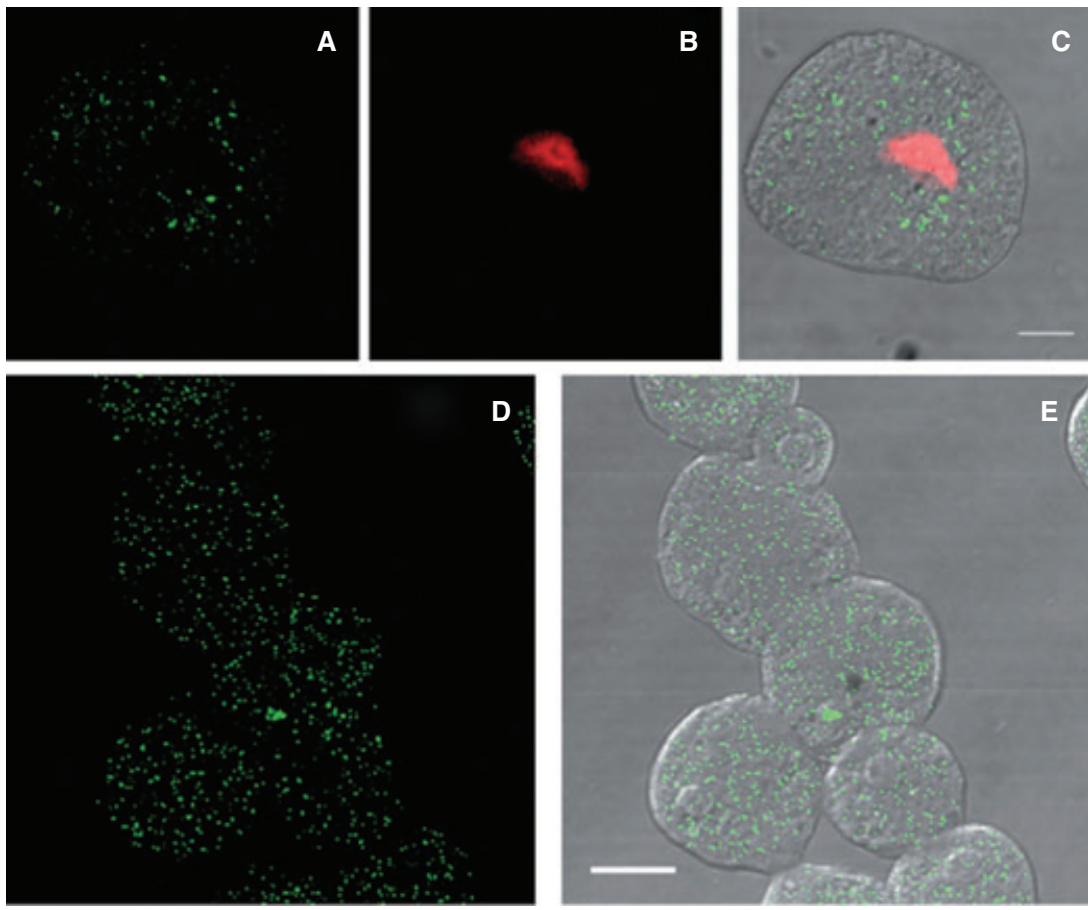

F $22 \mathrm{kDa}$

$22 \mathrm{kDa}$

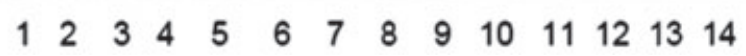

Fig. 5. Compartmentalized distribution of amoebal rubrerythrin.

A-E. Fixed parasite trophozoites were incubated with anti-TvRbr antibodies (A and D) and post-treated with propidium iodide to identify nuclei (B). (C and E) Superimposed DIC images showing individual cells. Scale bars: $5 \mu \mathrm{m}(\mathrm{C}), 10 \mu \mathrm{m}(\mathrm{E})$.

F. E. histolytica cell-free extracts were separated by sucrose density gradient centrifugation as described in Experimental procedures. Fractions were then analysed by Western blotting using the anti-TvRbr antibodies. Fraction number (fractions collected from bottom of tube), antibody specificity and the molecular mass of identified protein bands are indicated. compares favourably with the volume occupied by the amoebal nucleus $(-1.5 \%)$, suggesting that although mitosomes may no longer be involved in energy metabolism, they still likely represent important sites of metabolic flow in this parasite.

\section{Discussion}

Accumulating evidence indicates that mitochondria, mitosomes and hydrogenosomes are contemporary evolutionary endpoints of the original mitochondrial endosymbiont. In an extreme example of reductive organelle evolution E. histolytica has replaced its original mitochondrial Isc system of Fe-S cluster assembly with an analogous bacterial Nif system acquired by lateral gene transfer. The essential nature of compartmentalized Fe-S cluster assembly suggests that loss of the mitochondrial Isc system could only have taken place once the bacterial Nif system had been reliably adopted to function within E. histolytica mitosomes. That bacterial proteins other than those from the original mitochondrial endosymbiont were recruited during the course of evolution to function within mitochondria is reflected in the chimeric nature of the mitochondrial proteome (Kurland and Andersson, 2000; Esser et al., 2004). The presence in Entamoeba mitosomes of bacterial-type Fe-S cluster assembly proteins takes this observation a step further and demon- strates that analogous protein replacement has also played an active role in the evolution of mitochondrionrelated organelles.

The predominantly cytosolic distribution of bacterialtype Fe-S cluster assembly proteins NifS and NifU in Entamoeba contrasts with the predominantly mitochondrial localization of Fe-S cluster assembly in other eukaryotes (Lill and Mühlenhoff, 2008). In human cells a very small proportion of the cysteine desulfurase Nfu1 and the scaffold protein Isu1 - both essential for Fe-S cluster assembly - are found in cytosol and nuclei. However, maturation of both mitochondrial and extramitochondrial Fe-S proteins is known to require Fe-S clusters assembled in mitochondria. These are exported from the organelle via the Atm1/Erv1 export system (Gerber et al., 2004; Lill and Mühlenhoff, 2008). In Entamoeba, Fe-S centres synthesized in the cytosol are unlikely to be available for the maturation of mitosomal Fe-S proteins. This would require a specific cytosol-to-mitosome Fe-S cluster import system equivalent to but of reverse flux to the Atm1/Erv1-mediated mitochondrial export system. That no homologues of such transport proteins have been found in the E. histolytica genome suggests that these vital cofactors are not transported through membranes in this organism (Loftus et al., 2005; Clark et al., 2007).

Instead, the recruitment of a significant proportion of the cellular Fe-S cluster assembly activity into Entam- 

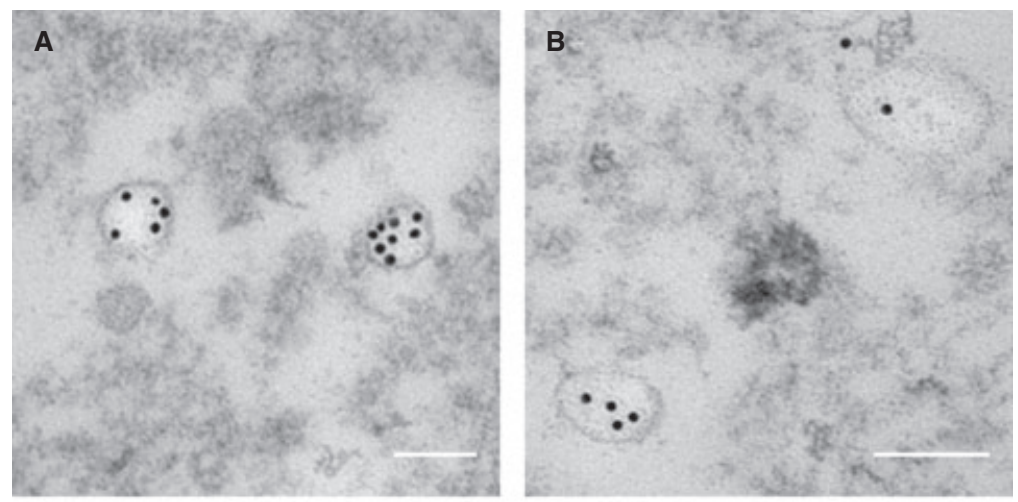

Fig. 6. Localization of E. histolytica rubrerythrin by immunoelectron microscopy. Fixed parasite trophozoites were embedded in resin and ultra-thin sections were mounted on grids before incubation with antibodies specific for TvRbr (A-D) or EhCpn60 (E). Double labelling using $20 \mathrm{~nm}$ gold particles for Cpn60 and $10 \mathrm{~nm}$ gold particles for Rbr is shown in F. Arrowheads in $\mathbf{c}$ indicate the organellar membranes. Scale bars: $100 \mathrm{~nm}$.
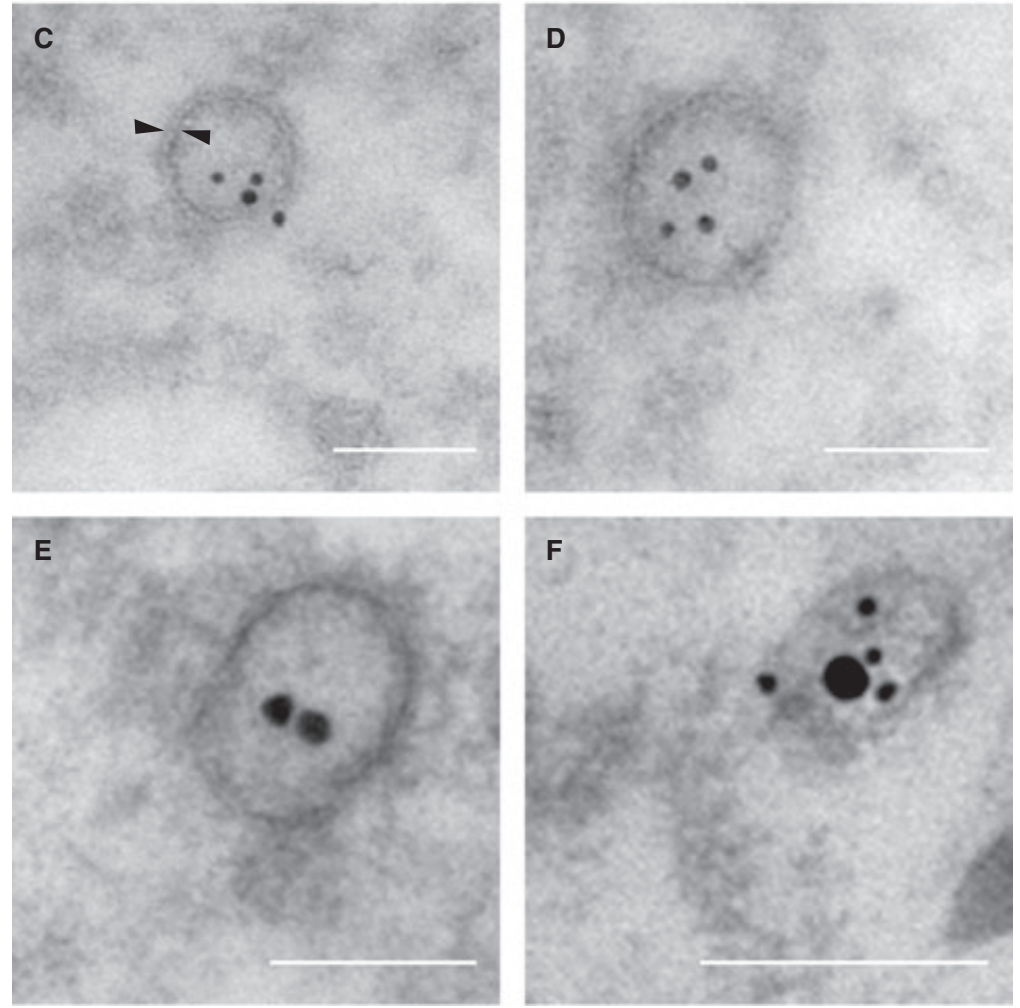

oeba mitosomes - estimated at approximately $17 \%$ of the total (the $1.7 \%$ average cell volume occupied by mitosomes is multiplied by the 10 -fold higher concentration of Nif proteins in mitosomes compared to cytosol) suggests that compartmentalized Fe-S cluster biosynthesis in E. histolytica is required for the in organello maturation of mitosomal proteins. At present it is unclear how bacterial proteins such as NifS, NifU and Rbr are imported into mitosomes as they lack typical amino-terminal targeting peptides to aid their passage across mitosomal membranes. However, protein import into mitosomes has been shown to proceed via both presequence-dependent and -independent mechanisms (Tovar et al., 1999; Dolezal et al., 2005; Regoes et al., 2005). Moreover, the natural predisposition of some bacterial proteins for mitochondrial targeting has been demonstrated (Lucattini et al., 2004).
Despite multiple efforts and the availability of the $E$. histolytica genome sequence no biological function had been assigned to amoebal mitosomes, until now (Loftus et al., 2005; Clark et al., 2007; Aguilera et al., 2008). For years it has been known that $E$. histolytica is capable of metabolizing oxygen with high affinity without the accumulation of peroxide (Reeves, 1984; Müller, 2003). In the absence of catalase and glutathione peroxidase it had been assumed that cytosolic peroxiredoxins and the thioredoxin/ thioredoxin reductase system were responsible for the removal of hydroperoxides from the cell (Choi et al., 2005; Davis et al., 2006; Arias et al., 2007). The identification of $\mathrm{Rbr}$ in mitosomes adds another dimension to the hydroperoxide detoxification capabilities of E. histolytica. Its functional Fe-S centres could allow Rbr to participate in the transfer of electrons from organic donors to oxygen 
via reduction of hydrogen peroxide, thus preventing its accumulation and toxicity inside these organelles. Whether oxygen metabolism occurs primarily in mitosomes or in the cytoplasm remains to be determined experimentally but the Rbr-dependent removal of toxic hydrogen peroxide in mitosomes could help the parasite survive the fluctuating low oxygen concentrations within the human intestine and, most importantly, the high concentrations of oxygen it encounters in the portal system during invasive amoebiasis (Stanley, 2003).

It has recently been reported that $\mathrm{Rbr}$ is upregulated in E. histolytica trophozoites exposed to oxidative stress clearly supporting the role of this mitosomal enzyme in hydroperoxide detoxification (Vicente et al., 2009). We conclude that Fe-S cluster biosynthesis and Fe-S proteinmediated oxygen detoxification are fundamental functions of E. histolytica mitosomes which together could have selected for the retention of the original mitochondrial endosymbiont in this microaerophilic eukaryote.

\section{Experimental procedures}

\section{Cell culturing and subcellular fractionation}

Entamoeba histolytica HM-1:IMSS parasites were cultured in YI-S medium supplemented with $15 \%$ adult bovine serum as described (Diamond et al., 1995). Axenically grown trophozoites were harvested by centrifugation, washed twice in PBS, resuspended in PBS/PI/E64 [PBS containing Protease inhibitor cocktail (Roche) and $50 \mu \mathrm{M}$ E64 (Sigma)] and lysed by freezethawing. After an initial centrifugation for $10 \mathrm{~min}$ at $1000 \mathrm{~g}$ the post-nuclear supernatant was subjected to ultracentrifugation at $100000 \mathrm{~g}$ for $1 \mathrm{~h}$ at $4^{\circ} \mathrm{C}$ to produce a high-speed supernatant (the cytosolic fraction) and a high-speed sediment (MMF) containing broken membranes and membrane-bounded organelles. The MMF was subsequently washed twice and resuspended in $\mathrm{PBS} / \mathrm{PI} / \mathrm{E} 64$. In some experiments both the post-nuclear supernatant and the MMF were further fractionated by density gradient centrifugation and fractions collected from the bottom of tubes. Samples were then subjected to Western blot analyses as described below. Protein concentrations were determined by the Bradford method (Bradford, 1976) using a commercial protein assay solution (Bio-Rad).

\section{SDS-PAGE, sample preparation and Western blotting}

Protein samples were mixed (1:1) with $2 \times$ Laemli sample buffer (Sigma) and heated at $96^{\circ} \mathrm{C}$ for $5 \mathrm{~min}$ prior to the SDS-PAGE. Ten microlitres of sample was loaded onto NuPAGE ${ }^{\circledR} 10 \%$ BISTRIS gels (Invitrogen) and separated at $150 \mathrm{~V}$. For Western blotting, proteins were transferred onto a nitrocellulose membrane (Bio-Rad) at $150 \mathrm{~V}$ for $1 \mathrm{~h}$. The membranes were blocked in $4 \%(\mathrm{w} / \mathrm{v})$ skimmed milk in TBS-T buffer (Tris-buffer saline with $0.1 \%$ Tween 20) for $1 \mathrm{~h}$ at room temperature and subsequently incubated at $4{ }^{\circ} \mathrm{C}$ overnight with primary antibodies (anti-TvRbr; 1:1000; anti-EhCpn60, anti-EhNifS, anti-EhNifU; all three 1:5000 in blocking solution). Following washing in TBS-T membranes were further incubated with secondary Immunopure ${ }^{\circledR}$ peroxidase- conjugated goat anti-rabbit antibody (Pierce; 1:20 000 in blocking solution) for $1 \mathrm{~h}$ at room temperature. Blots were developed using an ECL detection system (GE Healthcare).

\section{Immunofluorescence confocal microscopy}

Entamoeba trophozoites were fixed in $4 \%$ paraformaldehyde in PBS for $30 \mathrm{~min}$ at $37^{\circ} \mathrm{C}$, washed in PBS and permeabilized with $0.2 \%$ triton $\mathrm{X}-100$ in PBS. Cells were then attached to poly-Llysine-coated microscopic slides and exposed to blocking solution (3\% BSA in PBS) for $3 \mathrm{~h}$ at room temperature, before incubation with antigen-specific primary antibodies (diluted 1:200 in blocking solution) overnight at $4^{\circ} \mathrm{C}$. Control slides were incubated with pre-immune serum and/or blocking solution. Following $4 \times$ washing in PBS containing $0.2 \%$ Triton $X-100$ slides were incubated with secondary Alexa Fluor $\AA_{488}$ goat anti-rabbit antibody (1:500 in blocking solution, Invitrogen) for $1 \mathrm{~h}$ at $37^{\circ} \mathrm{C}$. Slides were further washed in PBS, mounted with 1:1 mixture of VectaShield and $0.4 \mu \mathrm{g} \mathrm{ml}^{-1}$ propidium iodide and observed under a laser scanning confocal microscope (Radiance 2100, Bio-Rad). Z-stacks (optical slices 0.5 or $1 \mu \mathrm{m}$ in thickness) were collected using Bio-Rad LaserSharp 2000 software and further processed using Adobe Photoshop V7.0 and/or ImageJ (http://rsb.info.nih.gov/ij/) software packages.

\section{Immunoelectron microscopy}

Entamoeba trophozoites were fixed in $4 \%$ paraformaldehyde in PBS for $30 \mathrm{~min}$ at $37^{\circ} \mathrm{C}$, washed in PBS and gradually dehydrated in ethanol (30-100\%), infiltrated and embedded in K4M resin. Thin sections (70-100 $\mathrm{nm}$ thick) were cut with a diamond knife and mounted onto gold grids. Immunogold labeling was done on grids floating on drops. The samples were blocked and incubated with single primary antibodies (anti-EhCpn60, antiTvRbr, anti-EhNifS or anti-EhNifU; all 1:50 and 1:200 dilutions pre-immune serum or blocking solution were used in control grids) followed with secondary goat anti-rabbit $\lg G$ conjugated to immunogold particles of either $10 \mathrm{~nm}$ or $20 \mathrm{~nm}$ in diameter (BB International; 1:100 dilution in blocking buffer). Double labeling experiments were carried out in the same way but with an additional paraformaldehyde vapor treatment step between antibody incubations as previously described (Mahendrasingam et al., 2003). Labeled samples were then post stained with uranyl acetate and lead citrate and observed under a Hitachi H-7600 transmission electron microscope. Digital images were collected and stored as TIFF files and further processed using Adobe Photoshop V7.0 and/or ImageJ (http://rsb.info.nih.gov/ij/) software packages.

\section{Immunoelectron microscopy quantification and calculations}

Labeling densities were estimated by counting the number of gold particles on electronic micrographs layered with square lattice grids of 20 and $100 \mathrm{~nm}$ in Adobe Photoshop. Labeling densities in cytosol represent the number of gold particles not associated with biological membranes per square micron on thin sections. Organelle labeling densities were estimated by first determining the average number of gold particles per labeled 
organelle (total number of gold particles associated with membrane-bounded organelles per square micron divided by the total number of gold-labeled organelles in the same area) and then dividing the resulting values by the area of an organelle $100 \mathrm{~nm}$ in diameter $\left(7.85 \times 10^{-3} \mu \mathrm{m}^{2}\right)$. In parallel calculations that take into account the thickness of the thin sections imaged (100 nm maximum) the average number of labeled organelles per square micron was multiplied by 10 multiplied by 10 , not by 100 to estimate the average number of organelles per cubic micron $\left(\mu \mathrm{m}^{3}\right)$. Resulting values were then multiplied by the total volume of an average spherical trophozoite of 20 microns in diameter $\left(4189 \mu \mathrm{m}^{3}\right)$ to estimate the average number of organelles per parasite trophozoite. Similarly, the volume occupied by an average spherical organelle of $100 \mathrm{~nm}$ in diameter $\left(5.2 \times 10^{-4} \mu \mathrm{m}^{3}\right)$ was multiplied by the total number of organelles in a cell to determine the overall volume occupied by labeled organelles. These values were then divided by the average volume of a trophozoite and multiplying by 100 to express them as percentages of cell volume. Standard equations for calculating the area of a circle $\left(\pi \times r^{2}\right)$ and the volume of a sphere $\left(4 / 3 \pi \times r^{3}\right)$ were used as appropriate.

\section{Enzymatic assays}

Affinity purified, recombinant E. histolytica and T. vaginalis $\mathrm{Rbr}$ were tested for NADPH-dependent peroxidase activity in reaction mixtures containing $25 \mu \mathrm{g} \mathrm{Rbr}, 100 \mu \mathrm{M}$ NADPH, $0.5 \mu \mathrm{M}$ ferredoxin : NADP ${ }^{+}$oxidoreductase from spinach, $0.5 \mu \mathrm{M}$ rubredoxin from C. pasteurianum, $250 \mu \mathrm{M} \mathrm{H}_{2} \mathrm{O}_{2}, 50 \mathrm{mM}$ MOPS $\mathrm{pH} 7.0,0.1 \mathrm{mM}$ EDTA, $20 \mathrm{mM}$ glucose, $0.1 \mathrm{U} \mathrm{ml}^{-1}$ glucose oxidase (all enzymes purchased from Sigma). Assays were covered with butanol and extinction at $340 \mathrm{~nm}$ was followed in a Genesys 10 UV photometer at room temperature.

Ferredoxin and pyruvate: ferredoxin oxidoreductase activities were monitored in cell-free extracts following reduction of cytochrome $c$ at $550 \mathrm{~nm}$ and of methyl viologen or nitroblue tetrazolium at $578 \mathrm{~nm}$ respectively. Extracts were prepared from 30 to $50 \mathrm{mg}$ of cell pellets suspended in $200 \mu \mathrm{l}$ of $50 \mathrm{mM}$ Tris- $\mathrm{HCl}$ buffer pH 9.0, 1 mM EDTA, 2.0 mM DTT, 15\% glycerol supplemented with protease inhibitors (E64 and Complete Mini Cocktail, Roche), sonicated and centrifuged at $10000 \mathrm{~g}$. The supernatant was collected and used in enzymatic reactions.

\section{Acknowledgements}

We thank Neil Sommerville for parasite culture and Patricia Goggin (Southhampton University) for advice and help with the initial electron microscopy work. The participation of Dr Gloria León-Avila and Mr Adhiraj Chakrabarty in preliminary work related to this project is acknowledged. This work was supported by a project grant from the BBSRC (BB/C507145) to J.T.

\section{References}

Aguilera, P., Barry, T., and Tovar, J. (2008) Entamoeba histolytica mitosomes: organelles in search of a function. Exp Parasitol 118: 10-16.

Ali, V., Shigeta, Y., Tokumoto, U., Takahashi, Y., and Nozaki, T. (2004) An intestinal parasitic protist, Entamoeba histolytica, possesses a non-redundant nitrogen fixation-like system for iron-sulfur cluster assembly under anaerobic conditions. J Biol Chem 279: 16863-16874.

Arias, D.G., Gutierrez, C.E., Iglesias, A.A., and Guerrero, S.A. (2007) Thioredoxin-linked metabolism in Entamoeba histolytica. Free Radic Biol Med 42: 1496-1505.

Bakatselou, C., Kidgell, C., and Clark, C.G. (2000) A mitochondrial-type hsp70 gene of Entamoeba histolytica. Mol Biochem Parasitol 110: 177-182.

Bakatselou, C., Beste, D., Kadri, A.O., Somanath, S., and Clark, C.G. (2003) Analysis of genes of mitochondrial origin in the genus Entamoeba. J Eukaryot Microbiol 50: 210-214.

Beinert, H. (2000) Iron-sulfur proteins: ancient structures, still full of surprises. J Biol Inorg Chem 5: 2-15.

Beinert, H., Holm, R.H., and Munck, E. (1997) Iron-sulfur clusters: nature's modular, multipurpose structures. Science 277: 653-659.

Bradford, M.M. (1976) A rapid and sensitive method for the quantitation of microgram quantities of protein utilizing the principle of protein-dye binding. Anal Biochem 72: 248254.

Chan, K.W., Slotboom, D.J., Cox, S., Embley, T.M., Fabre, O., van der Giezen, M. et al. (2005) A novel ADP/ATP transporter in the mitosome of the microaerophilic human parasite Entamoeba histolytica. Curr Biol 15: 737-742.

Choi, M.H., Sajed, D., Poole, L., Hirata, K., Herdman, S., Torian, B.E., et al. (2005) An unusual surface peroxiredoxin protects invasive Entamoeba histolytica from oxidant attack. Mol Biochem Parasitol 143: 80-89.

Clark, C.G., Alsmark, U.C., Tazreiter, M., Saito-Nakano, Y., Ali, V., Marion, S., et al. (2007) Structure and content of the Entamoeba histolytica genome. Adv Parasitol 65: 51-190.

Coulter, E.D., and Kurtz, D.M., Jr (2001) A role for rubredoxin in oxidative stress protection in Desulfovibrio vulgaris: catalytic electron transfer to rubrerythrin and two-iron superoxide reductase. Arch Biochem Biophys 394: 7686.

Coulter, E.D., Shenvi, N.V., and Kurtz, D.M., Jr (1999) NADH peroxidase activity of rubrerythrin. Biochem Biophys Res Commun 255: 317-323.

Craig, E.A., and Marszalek, J. (2002) A specialized mitochondrial molecular chaperone system: a role in formation of Fe/S centers. Cell Mol Life Sci 59: 1658-1665.

Davis, P.H., Zhang, X., Guo, J., Townsend, R.R., and Stanley, S.L., Jr (2006) Comparative proteomic analysis of two Entamoeba histolytica strains with different virulence phenotypes identifies peroxiredoxin as an important component of amoebic virulence. Mol Microbiol 61: 15231532.

Diamond, L.S., Clark, C.G., and Cunnick, C.C. (1995) YI-S, a casein-free medium for axenic cultivation of Entamoeba histolytica, related Entamoeba, Giardia intestinalis and Trichomonas vaginalis. J Eukaryot Microbiol 42: 277278.

Dolezal, P., Smíd, O., Rada, P., Zubácová, Z., Suták, R., Bursać, D., et al. (2005) Giardia mitosomes and trichomonad hydrogenosomes share a common mode of protein targeting. Proc Natl Acad Sci USA 102: 1092410929.

Embley, T.M. (2006) Multiple secondary origins of the 
anaerobic lifestyle in eukaryotes. Philos Trans $R$ Soc Lond B Biol Sci 361: 1055-1067.

Embley, T.M., Finlay, B.J., Dyal, P.L., Hirt, R.P., Wilkinson, M., and Williams, A.G. (1995) Multiple origins of anaerobic ciliates with hydrogenosomes within the radiation of aerobic ciliates. Proc $R$ Soc Lond B Biol Sci 262: 8793.

Embley, T.M., van der Giezen, M., Horner, D.S., Dyal, P.L., Bell, S., and Foster, P.G. (2003) Hydrogenosomes, mitochondria and early eukaryotic evolution. IUBMB Life 55: 387-395.

Esser, C., Ahmadinejad, N., Wiegand, C., Rotte, C., Sebastiani, F., Gelius-Dietrich, G., et al. (2004) A genome phylogeny for mitochondria among alpha-proteobacteria and a predominantly eubacterial ancestry of yeast nuclear genes. Mol Biol Evol 21: 1643-1660.

Frazzon, J., and Dean, D.R. (2003) Formation of iron-sulfur clusters in bacteria: an emerging field in bioinorganic chemistry. Curr Opin Chem Biol 7: 166-173.

Gerber, J., Neumann, K., Prohl, C., Mühlenhoff, U., and Lill, R. (2004) The yeast scaffold proteins Isu1p and Isu2p are required inside mitochondria for maturation of cytosolic Fe/S proteins. Mol Cell Biol 24: 4848-4857.

Ghosh, S., Field, J., Rogers, R., Hickman, M., and Samuelson, J. (2000) The Entamoeba histolytica mitochondrionderived organelle (crypton) contains double-stranded DNA and appears to be bound by a double membrane. Infect Immun 68: 4319-4322.

van der Giezen, M., Cox, S., and Tovar, J. (2004) The ironsulphur cluster assembly genes iscS and iscU of Entamoeba histolytica were acquired by horizontal gene transfer. BMC Evol Biol 4: 7.

van der Giezen, M., Tovar, J., and Clark, C.G. (2005a) Mitochondrion-derived organelles in protists and fungi. Int Rev Cytol 244: 177-227.

van der Giezen, M., León-Avila, G., and Tovar, J. (2005b) Characterization of chaperonin 10 (Cpn10) from the intestinal human pathogen Entamoeba histolytica. Microbiology 151: 3107-3115.

Gill, E.E., Diaz-Trivino, S., Barbera, M.J., Silberman, J.D., Stechmann, A., Gaston, D., et al. (2007) Novel mitochondrion-related organelles in the anaerobic amoeba Mastigamoeba balamuthi. Mol Microbiol 66: 13061320.

Goldberg, A.V., Molik, S., Tsaousis, A.D., Neumann, K., Kuhnke, G., Delbac, F., et al. (2008) Localization and functionality of microsporidian iron-sulphur cluster assembly proteins. Nature 452: 624-628.

Jin, S., Kurtz, D.M., Jr., Liu, Z.J., Rose, J., and Wang, B.C. (2002) X-ray crystal structures of reduced rubrerythrin and its azide adduct: a structure-based mechanism for a non-heme diiron peroxidase. J Am Chem Soc 124: 98459855.

Katinka, M.D., Duprat, S., Cornillot, E., Metenier, G., Thomarat, F., Prensier, G., et al. (2001) Genome sequence and gene compaction of the eukaryote parasite Encephalitozoon cuniculi. Nature 414: 450-453.

Kurland, C.G., and Andersson, S.G. (2000) Origin and evolution of the mitochondrial proteome. Microbiol Mol Biol Rev 64: 786-820.

León-Avila, G., and Tovar, J. (2004) Mitosomes of Entam- oeba histolytica are abundant mitochondrion-related remnant organelles that lack a detectable organellar genome. Microbiology 150: 1245-1250.

Lill, R., and Kispal, G. (2000) Maturation of cellular Fe-S proteins: an essential function of mitochondria. Trends Biochem Sci 25: 352-356.

Lill, R., and Mühlenhoff, U. (2008) Maturation of iron-sulfur proteins in eukaryotes: mechanisms, connected processes, and diseases. Annu Rev Biochem 77: 1-32.

Loftus, B., Anderson, I., Davies, R., Alsmark, U.C., Samuelson, J., Amedeo, P., et al. (2005) The genome of the protist parasite Entamoeba histolytica. Nature 433: 865-868.

Loiseau, L., Ollagnier-d., e-Choudens, S., Nachin, L., Fontecave, M., and Barras, F. (2003) Biogenesis of Fe-S cluster by the bacterial Suf system: SufS and SufE form a new type of cysteine desulfurase. J Biol Chem 278: 3835238359.

Lucattini, R., Likic, V.A., and Lithgow, T. (2004) Bacterial proteins predisposed for targeting to mitochondria. Mol Biol Evol 21: 652-658.

Mahendrasingam, S., Wallam, C.A., and Hackney, C.M. (2003) Two approaches to double post-embedding immunogold labeling of freeze-substituted tissue embedded in low temperature Lowicryl HM20 resin. Brain Res Protoc 11: 134-141.

Morrison, H.G., McArthur, A.G., Gillin, F.D., Aley, S.B., Adam, R.D., Olsen, G.J., et al. (2007) Genomic minimalism in the early diverging intestinal parasite Giardia lamblia. Science 317: 1921-1926.

Müller, M. (2000) A mitochondrion in Entamoeba histolytica? Parasitol Today 16: 368-369.

Müller, M. (2003) Energy metabolism. Part I: anaerobic protozoa. In Molecular Medical Parasitology. Marr, J., Nilsen, T., and Komuniecki, R. (eds). London: Academic Press, pp. 125-139.

Outten, F.W., Wood, M.J., Munoz, F.M., and Storz, G. (2003) The SufE protein and the SufBCD complex enhance SufS cysteine desulfurase activity as part of a sulfur transfer pathway for Fe-S cluster assembly in Escherichia coli. $J$ Biol Chem 278: 45713-45719.

Pütz, S., Gelius-Dietrich, G., Piotrowski, M., and Henze, K. (2005) Rubrerythrin and peroxiredoxin: two novel putative peroxidases in the hydrogenosomes of the microaerophilic protozoon Trichomonas vaginalis. Mol Biochem Parasitol 142: 212-223.

Rees, D.C., and Howard, J.B. (2000) Nitrogenase: standing at the crossroads. Curr Opin Chem Biol 4: 559-566.

Reeves, R.E. (1984) Metabolism of Entamoeba histolytica Schaudinn, 1903. Adv Parasitol 23: 105-142.

Regoes, A., Zourmpanou, D., León-Avila, G., van der Giezen, M., Tovar, J., and Hehl, A.B. (2005) Protein import, replication, and inheritance of a vestigial mitochondrion. $J$ Biol Chem 280: 30557-30563.

Stanley, S.L., Jr (2003) Amoebiasis. Lancet 361: 1025-1034. Suták, R., Dolezal, P., Fiumera, H.L., Hrdý, I., Dancis, A., Delgadillo-Correa, M., et al. (2004) Mitochondrial-type assembly of FeS centers in the hydrogenosomes of the amitochondriate eukaryote Trichomonas vaginalis. Proc Natl Acad Sci USA 101: 10368-10373.

Takahashi, Y., and Tokumoto, U. (2002) A third bacterial system for the assembly of iron-sulfur clusters with 
homologs in archaea and plastids. J Biol Chem 277: 28380-28383.

Tovar, J. (2007) Mitosomes of parasitic protozoa: biology and evolutionary significance. In Origin of Mitochondria and Hydrogenosomes. Martin, W.F., and Müller, M. (eds). Heidelberg: Springer-Verlag, pp. 277-300.

Tovar, J., Fischer, A., and Clark, C.G. (1999) The mitosome, a novel organelle related to mitochondria in the amitochondrial parasite Entamoeba histolytica. Mol Microbiol 32: 1013-1021.

Tovar, J., León-Avila, G., Sánchez, L.B., Sutak, R., Tachezy, J., van der Giezen, M., et al. (2003) Mitochondrial remnant organelles of Giardia function in iron-sulphur protein maturation. Nature 426: 172-176.

Vicente, J.B., Ehrenkaufer, G.M., Saraiva, L.M., Teixeira, M., and Singh, U. (2009) Entamoeba histolytica modulates a complex repertoire of novel genes in response to oxidative and nitrosative stresses: implications for amebic pathogenesis. Cell Microbiol 11: 51-69.

Weinberg, M.V., Jenney, F.E., Jr., Cui, X., and Adams, M.W. (2004) Rubrerythrin from the hyperthermophilic archaeon Pyrococcus furiosus is a rubredoxin-dependent, ironcontaining peroxidase. J Bacteriol 186: 7888-7895.

\section{Supporting information}

Additional Supporting Information may be found in the online version of this article:

Fig. S1. Immunoblot analysis of ectopic expression of epitopeor GFP-tagged NifS and NifU. Blots were reacted with antiEhNifS (A), anti-EhNifU (B), anti-Myc (C) or anti-HA antibody (D). Lanes are: 1, molecular markers; 2, Myc control transformant; 3, Myc-NifS; 4, HA control; 5, HA-NifU; 6, MycGFP; 7, MycGFPNifU; 8, Myc-NifS/HA-NifU; 9, Myc-NifS/MycGFP-NifU.

Fig. S2. Growth kinetics of the wild-type and transgenic E. histolytica lines. Trophozoites from wild-type, Myc- and HA-control transformants as well as HA-NifU, Myc-NifS and Myc-NifS/HANifU transgenic parasite lines were seeded at initial densities of $1 \times 10^{4} \mathrm{ml}^{-1}$ in $6 \mathrm{ml}$ glass tubes containing BI-S-33 growth medium. Cells were counted daily using a hemocytometer; mean values and standard errors of the mean are plotted.

Please note: Wiley-Blackwell are not responsible for the content or functionality of any supporting materials supplied by the authors. Any queries (other than missing material) should be directed to the corresponding author for the article. 